\title{
Myospila bekilyana (Séguy) (Diptera, Muscidae, Mydaeinae): redescription of male and female and description of terminalia
}

\author{
Márcia S. Couri ${ }^{1,2}$ \\ ${ }^{1}$ Museu Nacional, Universidade Federal do Rio de Janeiro, Quinta da Boa Vista, 20940-040 Rio de Janeiro-RJ, Brasil. mcouri@terra.com.br \\ ${ }^{2}$ Research Fellow Conselho Nacional de Desenvolvimento Científico e Tecnológico, CNPq.
}

\begin{abstract}
Myospila bekilyana (Séguy) (Diptera, Muscidae, Mydaeinae): redescription of male and female and description of terminalia. Myospila bekilyana Séguy (Diptera, Muscidae, Mydaeainae) is an endemic species from Madagascar. Since the original description, the species has been referred in literature only in catalogues. A material recently collected by the Madagascar Arthropod Biodiversity project, mostly deposited at the California Academy of Sciences collection enabled the exam of a large series of this species. Male and female are herein redescribed and the terminalia of both sexes are described and illustrated for the first time. The paper also records Myospila as a new host for Stylogaster Macquart (Diptera, Conopidae).
\end{abstract}

KEYWORDS. Madagascar; morphology; parasite; taxonomy.

RESUMO. Myospila bekilyana (Séguy) (Diptera, Muscidae, Mydaeinae): redescrições do macho e da fêmea e descrição da terminália. Myospila bekilyana Séguy (Diptera, Muscidae, Mydaeainae) é uma espécie endêmica de Madagascar. Desde a sua descrição original, tem sido referida na literatura apenas em catálogos. Material recentemente coletado pelo "Madagascar Arthropod Biodiversity project", e quase em sua totalidade depositado na coleção da "California Academy of Sciences" permitiu o exame de uma larga série desta espécie. Macho e fêmea são redescritos e a terminália de ambos os sexos é descrita e ilustrada pela primeira vez. O artigo também registra Myospila como novo hospedeiro de Stylogaster Macquart (Diptera, Conopidae).

PALAVRAS-CHAVE. Madagascar; morfologia; parasita; taxonomia.

Myospila bekilyana (Diptera, Muscidae, Mydaeainae) was originally described by Séguy (1938) under the genus Dimorphia. The author compared the new species with Dimorphia flavicornis Macquart (synonym of Dimorphia cognata Robineau-Desvoidy), giving a brief compared description. Male and female were described from material collected in Bekily (Madagascar). Pont (1980) transferred the species to Myospila Rondani.

M. bekilyana is one of three Myospila species recorded from Madagascar, and the only one endemic to this region. Myospila paradoxalis (Stein in Becker, 1903) is also recorded from Egypt, Ghana, Madagascar, Nigeria and South Africa and Myospila lenticeps (Thomson, 1869) is widespread in Africa, occurring also in Principe Is. and in the Oriental region.

Couri et al. (2006) presented a key to the Madagascan Myospila species, where M. bekilyana can be recognized by the reddish yellow postpronotum contrasting with the rest of the brown grayish mesonotum, abdomen with faint lateral spots and yellow femora.

The aim of the present contribution is to give a detailed redescription of $M$. bekilyana with the description and illustrations, for the first time, of male and female terminalia. It is also included the first record of M. bekilyana as a host of Stylogaster Macquart.

\section{MATERIALAND METHODS}

The material studied was collected by the Madagascar Arthropod Biodiversity Project from 2001 to 2005. More details on this project can be seen in Couri et al. (2006) as well as the full list of material of $M$. bekilyana examined. The material listed here, including the dissected specimens, corresponds to the specimens deposited in Museu Nacional, Rio de Janeiro (Brasil) collection.

The dissected terminalia were clarified in a solution of $10 \%$ potassium hydroxide for 24 hours. They were then dissected in glycerol, drawn, stored in a microtube with glycerol, and attached to the pin bearing the specimen.

\section{RESULTS ANDDISCUSSION}

Myospila bekilyana (Séguy, 1938)

(Figs. 1-6)

Dimorphia bekilyana Séguy, 1938: 118.

Myospila bekilyana: Pont, 1980: 746 (catalogue, comb. n.); Couri et al. (2006): 867 (key).

Male. Body length: $8.0 \mathrm{~mm}$; wing: $8.5 \mathrm{~mm}$

General coloration. Ground color brown with grey pollinosity; frons, fronto-orbital plate and gena silver pollinose. Antenna brown, scape yellowish; arista and palpus brown. Dorsum of mesonotum with 2 brown vittae between the dorsocentral rows of setae and acrostichals reaching middle length of scutum, 2 other lateral vittae beginning on notopleuron and also reaching the middle length of scutum. Post-pronotum reddish yellow. Calypters yellowish and halter yellow. Wing clear. All legs yellow. Abdomen brown with grey pollinosity; abdomen of male with tergite $1+2$ yellow, tergite 3 
with yellow lateral areas close to anterior margin, followed by faint brown areas, tergite 4 with two faint brown lateral spots and tergite 5 yellow on apical two thirds (Fig. 1). Abdomen of female brown with grey pollinosity, tergite $1+2$ with two faint lateral yellow areas close to the anterior margin, tergite 3 with two lateral faint brown spots, tergite 4 with no marks and tergite 5 yellow on apical two thirds (Fig. 2).

Head. Holoptic. Frontal row with 6 pairs of setae, the one closer to lunule longer. Inner and outer vertical setae short. Ocelar setae long. Antenna short, inserted below mid level of eyes; with flagellomere measuring about 2.2 times the pedicel length. Arista with long plumae. Palpus filiform. Cheek straight. Vibrissa long, inserted at oral margin.

Thorax. Dorsocentrals 2:4; acrostichals 0:1;2 postpronotal; 1 presutural; 1 intralar; prealar present but weak, 2 supraalars; 2 postsupra-alars. Notopleuron with two setae, similar in size. Scutellum with one medium-sized pair of basal setae, one sub-basal and one apical, the last two ones longer and similar in size. Prosternum bare. Anepisternum with a row of about 6 setae. Katepisternals 2:2, the infero-anterior ciliform. Katepimeron bare. Inferior calypter about 2.2 longer than the superior one. Wing veins $\mathrm{R}_{4+5}$ and $\mathrm{M}_{1+2}$ almost parallel at apex. Vein $R_{4+5}$ with a few setulae at base on both surfaces. Legs with fore femur with a complete posteroventral and posterodorsal row of setae; fore tibia with a long preapical seta; claws and pulvilli developed; mid femur with 3-4 sparse ventral setae on basal third; posterior surface with 3 preapical setae; mid tibia with 2 posterior seta on middle third and with a very long and strong ventral apical seta; hind femur with a complete anterodorsal row of similar setae, anteroventral surface with a complete row of setae, the apical 7-8 stronger; hind tibia with one sub-median anteroventral, one median anterodorsal, and apical setae on anterodorsal, dorsal and ventral surfaces.

Abdomen. Tergite 3 with 2 pairs of long lateral setae; tergite 4 with a row of 6 long apical setae and tergite 5 with a discal and an apical row, both with 6 long setae (Fig. 1). Sternite 5 with medium-sized setae in almost all extension, and two long on each posterior lobe (Fig. 3).

Terminalia. Cercal plate rectangular, with a deep anterior incision and medium-sized setae on almost all extension; surstyli large and longer than cercal plate (Fig. 4). Aedeagus as in Fig. 5.

Female. Body length: $8.0 \mathrm{~mm}$; wing: $8.5 \mathrm{~mm}$

Similar to male, differing as follows: distance of eyes at vertex about one third of head width; ocellar triangle with a silver mark reaching lunule; anteroventral row of setae on hind femur with only the last 4-5 stronger. Abdomen with tergite 5 with discal and apical rows, both with 4 long setae.

Ovipositor. Mydaea-type, as in Fig. 6.

Comments. The male nr. 3010476 shows, just behind the postpronotum, an egg of Stylogaster Macquart (Diptera, Conopidae). This genus is known from five species in Madagascar. Couri \& Pont (2006) recorded seven new muscid hosts and the present one is also new, being the first for the genus Myospila.

Received 03/05/2007; accepted 13/02/2008

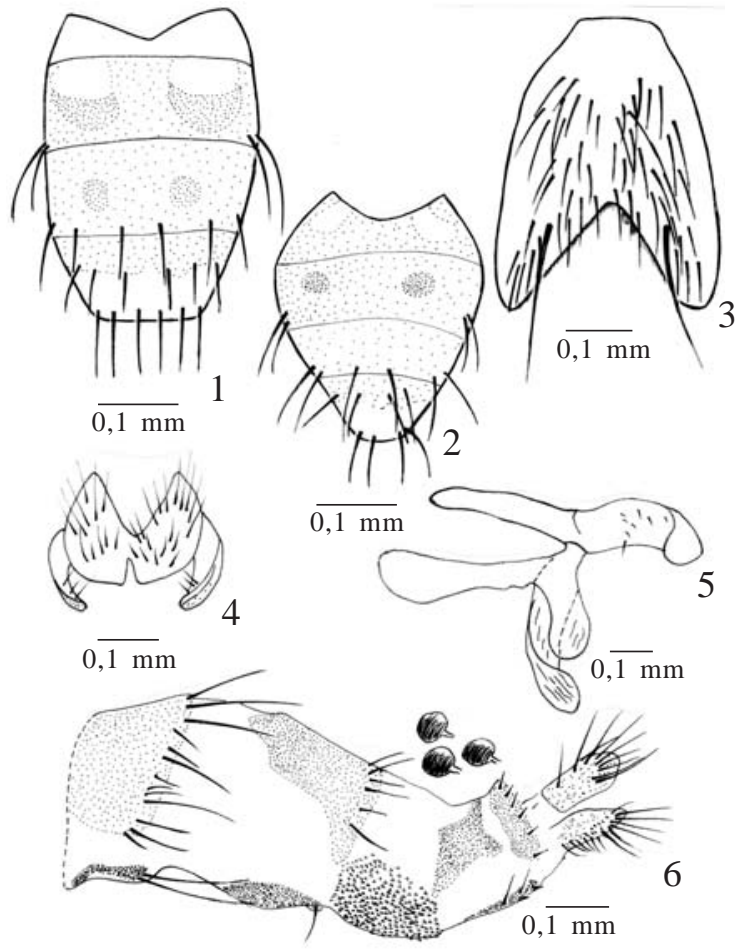

Figs. 1-6. Myospila bekilyana (Séguy). 1, Male, abdomen; 2, Female, abdomen; 3, Male, sternite 5; 4, Male, cercal plate and surstyli, dorsal view; 5, Male, aedeagus, lateral view; 6, Female, ovipositor, lateral view and spermathecae.

Material Examined. MADAGASCAR: Toliara Province: Antafoky, elev. $70 \mathrm{~m}, 23^{0} 31^{\prime} 38^{\prime}$ 'S $44^{0} 5^{\prime} 15^{\prime}$ 'E, Malaise trap, gallery forest at edge of marsh near road, 22-29 May 2002, Frontier Wilderness Project, collection code: MGF031, 1 female (dissected), CASENT 3009018. Fianarantsoa Province: Parc National Ranomafana, Belle Vue at Talatakely, elev. $1020 \mathrm{~m}, 21^{\circ} 15.99^{\prime} \mathrm{S} 47^{\circ} 25.21^{\prime}$, Malaise trap, secondary tropical forest, 28 January - 4 February 2002, R. Harin'Hala, collection code: MA-02-09C-14, 1 male, CASENT 3010476; Parc National d'Isalo, Ambovo Springs, $29.3 \mathrm{~km}, 4^{0} \mathrm{~N}$ Ranohira, elev. $990 \mathrm{~m}$, $22^{\circ} 17^{\prime} 54^{\prime \prime} \mathrm{S} 45^{\circ} 21^{\prime} 06^{\prime \prime} \mathrm{E}$, pitfall trap in Uapaca woodland, 9-14 February 2003, Fisher, Griswold et al., collection code: BLF7649, 1 male (dissected), CASENT 3009114.

Acknowledgments. I am grateful to all staff at the Department of Entomology of the California Academy of Sciences (San Francisco, California) for the donation of the examined material to Museu Nacional, UFRJ collection. I also acknowledge Conselho Nacional de Desenvolvimento Científico e Tecnológico (CNPq) for the research grant (process number 300370/2004-0) and financial support (process number 473115/2006).

\section{REFERENCES}

Couri, M. S. \& A. C. Pont. 2006. Eggs of Stylogaster Macquart (Diptera: Conopidae) on Madagascan Muscids (Diptera: Muscidae). Proceedings of the California Academy of Sciences 57: 473-478.

Couri, M. S.; A. C. Pont \& N. D. Penny. 2006. Muscidae (Diptera) from Madagascar: identification keys, descriptions of new species, and new records. Proceedings of the California Academy of Sciences 57: 799-923.

Séguy, E. 1938. Note sur les Anthomyiides (Muscidae). 12e note. Encyclopédie Entomologique (BII), Diptera 9: 109-120.

Pont, A. C. 1980. Family Muscidae. p. 721-761. In: R. W. Crosskey (ed.). Catalogue of the Diptera of the Afrotropical Region. British Museum (Natural History), London, UK. 